\title{
A reduced-order extrapolating Crank-Nicolson finite differ- ence scheme for the Riesz space fractional order equations with a nonlinear source function and delay
}

\author{
Yanhua Cao ${ }^{\mathrm{a}}$, Zhendong $\mathrm{Luo}^{\mathrm{b}, *}$ \\ a School of Sciences, East China Jiaotong University, Nanchang 330013, China. \\ ${ }^{b}$ School of Mathematics and Physics, North China Electric Power University, Beijing 102206, China.
}

Communicated by D. Baleanu

\begin{abstract}
This article mainly studies the order-reduction of the classical Crank-Nicolson finite difference (CNFD) scheme for the Riesz space fractional order differential equations (FODEs) with a nonlinear source function and delay on a bounded domain. For this reason, the classical CNFD scheme for the Riesz space FODE and the existence, stability, and convergence of the classical CNFD solutions are first recalled. And then, a reduced-order extrapolating CNFD (ROECNFD) scheme containing very few degrees of freedom but holding the fully second-order accuracy for the Riesz space FODEs is established by means of proper orthogonal decomposition and the existence, stability, and convergence of the ROECNFD solutions are discussed. Finally, some numerical experiments are presented to illustrate that the ROECNFD scheme is far superior to the classical CNFD one and to verify the correctness of theoretical results. This indicates that the ROECNFD scheme is very effective for solving the Riesz space FODEs with a nonlinear source function and delay.
\end{abstract}

Keywords: Crank-Nicolson finite difference scheme, Riesz space fractional order differential equation, existence and stability as well as convergence, reduced-order extrapolating Crank-Nicolson finite difference scheme, proper orthogonal decomposition.

2010 MSC: 34K28, 65M12, 35R11, 34K37.

(C)2018 All rights reserved.

\section{Introduction}

Because the fractional order differential equations (FODEs) have been a research hot-spot in science and engineering in recent years (see, e.g., $[13,15,17])$, this article mainly studies the order-reduction of the classical Crank-Nicolson finite difference (CNFD) scheme for the Riesz space FODEs with a nonlinear source function and delay on a bounded domain.

For convenience and without loss of generality, we take into account the following FODEs with a

\footnotetext{
*Corresponding author

Email addresses: yanhuacao@yeah.net (Yanhua Cao), zhdluo@ncepu.edu.cn (Zhendong Luo)

doi: $10.22436 /$ jnsa.011.05.08
}

Received: 2018-01-25 Revised: 2018-02-16 Accepted: 2018-03-03 
nonlinear source function and delay on the bounded domain $[-\mathrm{s}, \mathrm{T}] \times[0, \mathrm{~L}]$ :

$$
\begin{aligned}
\frac{\partial u(t, x)}{\partial t} & =K(t, x) \frac{\partial^{\alpha} u(t, x)}{\partial|\alpha|^{\alpha}}+f(t, x, u(t, x), u(t-s, x)), \quad(t, x) \in(0, T) \times(0, L), \\
u(t, 0) & =u(t, L)=g(t), \quad t \in(0, T), \\
u(t, x) & =\varphi(t, x), \quad(t, x) \in[-s, 0] \times(0, L),
\end{aligned}
$$

where $K(t, x)$ is the dispersion coefficient, $1<\alpha \leqslant 2, s>0, g(t)$ is a given boundary value function, $\varphi(t, x)$ is a given initial function, and $f(t, x, u, v)$ on $D=:[0, T] \times[0, L] \times \mathbb{R} \times \mathbb{R}$ is a given continuous real-value function that satisfies the Lipschitz condition, i.e., there exist two non-negative real numbers $\beta_{1} \geqslant 0$ and $\beta_{2} \geqslant 0$ such that

$$
\left|f\left(t, x, u_{1}, v_{1}\right)-f\left(t, x, u_{2}, v_{2}\right)\right| \leqslant \beta_{1}\left|u_{1}-u_{2}\right|+\beta_{2}\left|v_{1}-v_{1}\right|, \quad \forall u_{1}, v_{1}, u_{2}, v_{2} \in \mathbb{R} .
$$

In addition, the above $\partial^{\alpha} u(t, x) / \partial|\alpha|^{\alpha}$ is known as the Riesz space fractional order derivative and defined by

$$
\frac{\partial^{\alpha} u(t, x)}{\partial|\alpha|^{\alpha}}=-\frac{1}{2 \cos \left(\frac{\alpha \pi}{2}\right) \Gamma(2-\alpha)} \frac{\partial^{2}}{\partial x^{2}}\left(\int_{0}^{x}(x-y)^{1-\alpha} u(t, y) d y+\int_{x}^{L}(y-x)^{1-\alpha} u(t, y) d y\right),
$$

where $\Gamma(\cdot)$ is the Euler gamma function. For convenience and without loss of generality, we assume that $g(t)=0$ in the following analysis.

The Riesz space FODEs (1.1) hold very important physical background, such as, which can be used to describe phenomena in seepage hydraulics groundwater hydraulics, groundwater dynamics, and fluid dynamics in porous media (see, e.g., $[3,8,27,32])$. However, they usually have no analytic solution so that they mainly depend on numerical solutions (see, e.g., $[12,14,16,26])$. In recently, a classical CNFD scheme for the equations (1.1) has developed in [35], but it includes many degrees of freedom (i.e., unknowns). Thus, due to the truncated error amassing in the calculating process, it would appear floating point overflow after computing some steps so that it can't gain desired results. Therefore, in the case of ensuring the classical CNFD numerical solutions with the desired accuracy, how to decrease the unknowns of the classical CNFD scheme so as to simplify calculation and retard the truncated error amassing in the calculating process is an urgently solved problem in the practical applications, which is main objective in this paper.

Proper orthogonal decomposition (POD) method is considered as an effective and feasible optimized technique to reduce the order of numerical models (see $[2,7,25,30])$. It is essentially to look for a series of orthogonal basis for the known data in the sense of least square. The POD method can greatly reduce the unknowns in the numeric models. It has been extensively used in analysis of signal together with pattern recognition (see [5]), statistical computation (see [9]), and computational fluid dynamics (see [29]). In recent years, it also has been successfully applied to the order-reduction for the Galerkin method (see, e.g., $[10,11])$, the finite element method (see, e.g., [18, 22]), the FD scheme (see, e.g., [24, 31]), finite volume element method (see, e.g., [21, 23]), and reduced basis methods (see, e.g., [1, 6, 28]) for PDEs. However, the most existing POD reduced order methods (see, e.g., $[1,2,5-7,9-11,18,21-25,28-31]$ ) are built by the POD basis formed with the classical solutions at the all time nodes on $[0, T]$, before repetitively computing the reduced order solutions at the same time nodes. As a matter of fact, they are some valueless repetitive calculations. In order to eliminate the repetitive calculations, some POD reduced order extrapolated FD schemes have been addressed (see, e.g., [19, 20, 33, 34]).

However, for all we know, there has not been any research that the reduced-order extrapolating CNFD (ROECNFD) scheme for the Riesz space FODEs with a nonlinear source function and delay is built by the POD method. Hence, in this article, we develop the ROECNFD scheme only containing very few unknowns via the POD technique. Particularly, we only utilize the classical CNFD solutions at the initial a few time nodes as the snapshots to generate the POD bases and build the ROECNFD scheme for seeking out the ROECNFD solutions at all time nodes. This is equivalent to utilizing the existing information (on the quite short time interval $\left[0, \mathrm{~T}_{0}\right], \mathrm{T}_{0} \ll \mathrm{T}$ ) to forecast the future physical law (on the time interval 
$\left.\left[\mathrm{T}_{0}, T\right]\right)$. In addition, we here use the error estimations to guide the selection of POD bases. Because the ROECNFD scheme holds simultaneously both advantages of the POD technique and the CNFD scheme easily operating and holding the fully second-order accuracy, it is development and improvement over the existing reduced order methods as the above-mentioned.

The plan for the remainder of this paper is as follows. In Section 2, we first recall the classical CNFD scheme for the Riesz space FODEs with a nonlinear source function and delay, and form snapshots by the initial few classical CNFD solutions. In Section 3, we obtain a set of POD bases associated with the snapshots and establish the ROECNFD scheme. In Section 4, we discuss the existence, stability, and convergence of the ROECNFD solutions and provide the flowchart for solving the ROECNFD scheme. In Section 5, we use some numerical experiments to show that the ROECNFD scheme is superior to the classical CNFD one and to verify that the results of numerical computations are consistent with the theoretical analysis and that the ROECNFD scheme is very efficient for solving the Riesz space FODEs with a nonlinear source function and delay because it can greatly reduce the degrees of freedom and alleviate the calculation load as well as save the CPU time-consuming and the storage requirements in the computational process. Section 6 finally provides the main conclusions and discussions.

\section{The classical CNFD scheme for the Riesz space FODEs with a nonlinear source function and delay}

In this section, we recall the classical CNFD scheme for the equations (1.1), which is presented in [35]. Let $N$ and $M$ be two positive integers, $\tau=T / N$ be the time step-size and $h=L / M$ be the spatial step-size. The classical CNFD scheme with the predictor-corrector for the equations (1.1) is stated as follows:

$$
\begin{aligned}
\bar{u}_{i}^{n}= & u_{i}^{n-1}+\gamma\left[\sum_{k=0}^{i+1} \omega_{k}^{(\alpha)} u_{i-k+1}^{n-1}+\sum_{k=0}^{M-i+1} \omega_{k}^{(\alpha)} u_{i+k-1}^{n-1}\right]+\tau f\left(t_{n}, x_{i}, u_{i}^{n-1}, u_{i}^{n-m}\right), \\
u_{i}^{n}= & u_{i}^{n-1}+\gamma\left[\sum_{k=0}^{i+1} \omega_{k}^{(\alpha)} u_{i-k+1}^{n-1}+\sum_{k=0}^{M-i+1} \omega_{k}^{(\alpha)} u_{i+k-1}^{n-1}\right]+\frac{\tau}{2} f\left(t_{n}, x_{i}, u_{i}^{n-1}, u_{i}^{n-m}\right) \\
& +\gamma\left[\sum_{k=0}^{i+1} \omega_{k}^{(\alpha)} \bar{u}_{i-k+1}^{n-1}+\sum_{k=0}^{M-i+1} \omega_{k}^{(\alpha)} \bar{u}_{i+k-1}^{n-1}\right]+\frac{\tau}{2} f\left(t_{n}, x_{i}, \bar{u}_{i}^{n-1}, \bar{u}_{i}^{n-m}\right),
\end{aligned}
$$

where $u_{i}^{n}$ 's are approximate solution of $u\left(t_{n}, x_{i}\right)(i=1,2, \ldots, M), \gamma=-\tau K /\left[2 h^{\alpha} \cos (\alpha \pi / 2)\right], \omega_{0}^{(\alpha)}=$ $\alpha g_{0}^{(\alpha)} / 2, \omega_{k}^{(\alpha)}=\alpha g_{k}^{(\alpha)} / 2+(2-\alpha) g_{k-1}^{(\alpha)} / 2, g_{0}^{(\alpha)}=1, g_{k}^{(\alpha)}=[1-(1+\alpha) / k] g_{k-1}^{(\alpha)}(k=1,2, \ldots)$.

The sequences $\left\{\omega_{k}^{(\alpha)}\right\}_{k=0}^{\infty}$ and $\left\{g_{k}^{(\alpha)}\right\}_{k=0}^{\infty}$ have the following properties (see, e.g., [17, 35]).

Lemma 2.1. When $1<\alpha \leqslant 2$, the sequences $\left\{\omega_{k}^{(\alpha)}\right\}_{k=0}^{\infty}$ and $\left\{g_{k}^{(\alpha)}\right\}_{k=0}^{\infty}$ satisfy

(1) $g_{0}^{(\alpha)}=1, g_{1}^{(\alpha)}=-\alpha, g_{2}^{(\alpha)}=\alpha(\alpha-1) / 2>0,1 \geqslant g_{2}^{(\alpha)} \geqslant g_{3}^{(\alpha)} \geqslant \cdots \geqslant 0, \sum_{k=0}^{\infty} g_{k}^{(\alpha)}=0$, and $\sum_{k=0}^{m} g_{k}^{(\alpha)}<0(m \geqslant 1)$

(2) $\omega_{0}^{(\alpha)}=\alpha / 2, \omega_{1}^{(\alpha)}=\left(2-\alpha-\alpha^{2}\right) / 2<0, \omega_{2}^{(\alpha)}=\alpha\left(\alpha^{2}+\alpha-4\right) / 4,1 \geqslant \omega_{3}^{(\alpha)} \geqslant \omega_{4}^{(\alpha)} \geqslant \cdots \geqslant 0$, $\sum_{k=0}^{\infty} \omega_{k}^{(\alpha)}=0$, and $\sum_{k=0}^{m} \omega_{k}^{(\alpha)}<0(m \geqslant 2)$.

Set

$$
\begin{aligned}
& \mathbf{A}=\left[\begin{array}{cccccc}
\omega_{1}^{(\alpha)} & \omega_{0}^{(\alpha)} & 0 & \cdots & 0 & 0 \\
\omega_{2}^{(\alpha)} & \omega_{1}^{(\alpha)} & \omega_{0}^{(\alpha)} & \cdots & 0 & 0 \\
\omega_{3}^{(\alpha)} & \omega_{2}^{(\alpha)} & \omega_{1}^{(\alpha)} & \cdots & 0 & 0 \\
\vdots & \vdots & \vdots & \ddots & \vdots & \vdots \\
\omega_{M-2}^{(\alpha)} & \omega_{M-3}^{(\alpha)} & \omega_{M-4}^{(\alpha)} & \cdots & \omega_{1}^{(\alpha)} & \omega_{0}^{(\alpha)} \\
\omega_{M-1}^{(\alpha)} & \omega_{M-2}^{(\alpha)} & \omega_{M-3}^{(\alpha)} & \cdots & \omega_{2}^{(\alpha)} & \omega_{1}^{(\alpha)}
\end{array}\right], F(\mathbf{V})=\left[\begin{array}{c}
f\left(t_{n}, x_{1}, v_{1}, u_{1}^{n-m}\right) \\
f\left(t_{n}, x_{2}, v_{2}, u_{2}^{n-m}\right) \\
f\left(t_{n}, x_{3}, v_{3}, u_{3}^{n-m}\right) \\
\vdots \\
f\left(t_{n}, x_{M-2}, v_{M-2,}, u_{M-2}^{n-m}\right) \\
f\left(t_{n}, x_{M-1}, v_{M-1}, u_{M-1}^{n-m}\right)
\end{array}\right] \\
& \mathbf{u}^{\mathrm{n}}=\left[\mathrm{u}_{1}^{\mathrm{n}}, \mathrm{u}_{2}^{\mathrm{n}}, \ldots, \mathrm{u}_{M-2}^{\mathrm{n}}, \mathrm{u}_{M-1}^{\mathrm{n}}\right]^{\top}, \quad \overline{\mathbf{u}}^{\mathrm{n}}=\left[\overline{\mathrm{u}}_{1}^{\mathrm{n}}, \overline{\mathrm{u}}_{2}^{\mathrm{n}}, \ldots, \overline{\mathrm{u}}_{M-2}^{\mathrm{n}}, \overline{\mathrm{u}}_{M-1}^{\mathrm{n}}\right]^{\top}, \quad \mathbf{D}=-\gamma\left[\mathbf{A}+\mathbf{A}^{\top}\right] .
\end{aligned}
$$


Thus, the CNFD scheme (2.1) can be rewritten as the following matrix form.

$$
\begin{aligned}
& \overline{\mathbf{U}}^{\mathrm{n}}=\mathbf{U}^{\mathrm{n}-1}+\mathrm{D} \mathbf{U}^{\mathrm{n}-1}+\tau \mathrm{F}\left(\mathbf{U}^{\mathrm{n}-1}\right) \\
& \mathbf{U}^{\mathrm{n}}=\mathbf{U}^{\mathrm{n}-1}+\frac{1}{2} \mathbf{D}\left(\mathbf{U}^{\mathrm{n}-1}+\overline{\mathbf{U}}^{\mathrm{n}-1}\right)+\frac{\tau}{2}\left[\mathbf{F}\left(\mathbf{U}^{\mathrm{n}-1}\right)+\mathbf{F}\left(\overline{\mathbf{U}}^{\mathrm{n}-1}\right)\right] .
\end{aligned}
$$

Further, the vector form CNFD scheme (2.2) can simplified as the following:

$$
\begin{aligned}
\mathbf{U}^{n}= & \mathbf{U}^{n-1}+\frac{1}{2} \mathbf{D}\left(2 \mathbf{U}^{n-1}+\mathbf{D U}^{n-1}+\tau F\left(\mathbf{U}^{n-1}\right)\right) \\
& +\frac{\tau}{2}\left[\mathbf{F}\left(\mathbf{U}^{n-1}\right)+\mathbf{F}\left(\mathbf{U}^{n-1}+\mathbf{D U}^{n-1}+\tau F\left(\mathbf{U}^{n-1}\right)\right)\right], n=1,2, \ldots, N,
\end{aligned}
$$

subject to the initial condition

$$
\mathbf{u}^{0}=\left[u_{1}^{0}, u_{2}^{0}, \ldots, u_{M-2}^{0}, u_{M-1}^{0}\right]^{\top}, \quad u_{i}^{0}=\varphi(0, i h), \quad u_{i}^{k}=\varphi(k \tau, i h), \quad i=1,2, \ldots, M-1, k<0 .
$$

Obviously, the vector form CNFD scheme (2.3) has a unique series of solution vectors $\left\{\mathbf{U}^{\mathfrak{n}}\right\}_{\mathfrak{n}=1}^{N}$. The following stability and convergence of the series of solution $\left\{\mathbf{U}^{\mathfrak{n}}\right\}_{\mathfrak{n}=1}^{N}$ have been provided in [35, Theorems 4.2 and 4.3$]$.

Theorem 2.2. When $\|\mathbf{I}+\mathbf{D}\|_{\infty} \leqslant 1$, the series of solution $\left\{\mathbf{U}^{\mathrm{n}}\right\}_{\mathfrak{n}=1}^{\mathrm{N}}$ for the CNFD scheme (2.3) is stable and convergent. Further, the errors between the series of solution $\left\{\mathbf{U}^{\mathrm{n}}\right\}_{n=1}^{N}$ for the CNFD scheme (2.3) and $\tilde{\mathbf{u}}\left(t_{n}\right)=$ $\left[u\left(t_{n}, x_{1}\right), u\left(t_{n}, x_{2}\right), \ldots, u\left(t_{n}, x_{M-1}\right)\right]^{\top}(n=1,2, \ldots, N)$ formed by the analytic solution for the Riesz space FODEs (1.1) are as follows:

$$
\max _{1 \leqslant i \leqslant n}\left\|\tilde{\mathbf{U}}\left(\mathrm{t}_{i}\right)-\mathbf{U}^{i}\right\|_{\infty}=\mathrm{O}\left(\tau^{2}, \mathrm{~h}^{2}\right), \quad \mathrm{n}=1,2, \ldots, \mathrm{N},
$$

where I represents the unit matrix, $\|\tilde{\mathcal{A}}\|_{\infty}=\max _{1 \leqslant i \leqslant m} \sum_{j=1}^{m}\left|a_{i, j}\right|\left(\right.$ for any matrix $\left.\tilde{A}=\left(a_{i, j}\right)_{m \times m}\right)$, and $\left\|\mathbf{U}^{i}\right\|_{\infty}=\max _{1 \leqslant j \leqslant M-1}\left|\mathbf{u}_{j}^{i}\right|\left(\right.$ for any $\left.\mathbf{U}^{i}=\left(u_{1}^{i}, u_{2}^{i}, \ldots, u_{M-1}^{i}\right)^{\top} \in \mathbb{R}^{M-1}\right)$.

Remark 2.3. It is easily known from Lemma 2.1 that the condition $\|\mathbf{I}+\mathbf{D}\|_{\infty} \leqslant 1$ is reasonable. Thus, so long as the time step-size $\tau$, the spatial step-size $h$, the coefficient of dispersion $K(t, x)$, the source function $f$, the initial function $\varphi$, and parameters $\alpha$ and $s$ are given, the series of solution $\left\{\mathbf{U}^{\mathrm{n}}\right\}_{\mathfrak{n}=1}^{\mathrm{N}}$ can be obtained by solving the vector form CNFD scheme (2.3). The first $l$ solution vectors $\mathbf{U}^{1}, \mathbf{U}^{2}, \ldots, \mathbf{U}^{l}(l \ll N)$ in the series of solution $\left\{\mathbf{U}^{\mathfrak{n}}\right\}_{\mathfrak{n}=1}^{N}$ for the CNFD scheme (2.3) are extracted to form a set of snapshots.

\section{Establishment of the ROEFD scheme}

\subsection{Fabrication and properties of POD basis}

For the first $l$ solution vectors $\mathbf{U}^{1}, \mathbf{U}^{2}, \ldots, \mathbf{U}^{\mathrm{l}}(\mathrm{l} \ll \mathrm{N})$ extracted in Section 2 , let $\mathbf{A}_{\mathfrak{u}}=\left(\mathbf{U}^{1}, \mathbf{U}^{2}, \ldots, \mathbf{U}^{\mathrm{l}}\right)$ (apparently $\left.\boldsymbol{A}_{\mathfrak{u}} \in \mathbb{R}^{(M-1) \times l}\right), \lambda_{j}>0\left(j=1,2, \cdots, r=: \operatorname{rank}\left(\boldsymbol{A}_{\mathfrak{u}}\right)\right)$ be the positive eigenvalues of $\boldsymbol{A}_{\mathfrak{u}} \boldsymbol{A}_{\mathfrak{u}}^{\top}$ arranged non-increasingly and $\mathbf{u}_{\mathfrak{u}}=\left(\boldsymbol{\phi}_{1}, \boldsymbol{\phi}_{2}, \cdots, \boldsymbol{\phi}_{\mathrm{r}}\right) \in \mathbb{R}^{(\mathrm{M}-1) \times \mathrm{r}}$ be the orthonormal eigenvectors of $\boldsymbol{A}_{\mathfrak{u}} \boldsymbol{A}_{\mathfrak{u}}^{\top}$ corresponding to the positive eigenvalues. Then, a POD basis $\boldsymbol{\Phi}=:\left(\boldsymbol{\phi}_{1}, \boldsymbol{\phi}_{2}, \cdots, \boldsymbol{\phi}_{\mathrm{d}}\right)(\mathrm{d} \leqslant \mathrm{r})$ attained from the initial $d$ vectors in $\boldsymbol{u}_{\mathfrak{u}}$ satisfies the following formula (see, e.g., $[24,31]$ ):

$$
\left\|\boldsymbol{A}_{\mathfrak{u}}-\boldsymbol{\Phi} \boldsymbol{\Phi}^{\top} \boldsymbol{A}_{\mathfrak{u}}\right\|_{2,2}=\sqrt{\lambda_{\mathrm{d}+1}},
$$

where $\left\|\boldsymbol{A}_{\mathfrak{u}}\right\|_{2,2}=\sup _{\boldsymbol{x} \in \mathbb{R}^{M-1}}\left\|\boldsymbol{A}_{\mathfrak{u}} \boldsymbol{x}\right\|_{2} /\|\boldsymbol{x}\|_{2}$ and $\|\boldsymbol{x}\|_{2}$ is the Euclidean norm of $\boldsymbol{x} \in \mathbb{R}^{M-1}$. Further, there hold

$$
\left\|\mathbf{U}^{\mathrm{n}}-\boldsymbol{\Phi} \boldsymbol{\Phi}^{\top} \mathbf{U}^{\mathrm{n}}\right\|_{2}=\left\|\left(\boldsymbol{A}_{\mathfrak{u}}-\boldsymbol{\Phi} \boldsymbol{\Phi}^{\top} \boldsymbol{A}_{\mathfrak{u}}\right) \varepsilon^{\mathfrak{n}}\right\|_{2} \leqslant\left\|\boldsymbol{A}_{\mathfrak{u}}-\boldsymbol{\Phi} \boldsymbol{\Phi}^{\top} \boldsymbol{A}_{\mathfrak{u}}\right\|_{2,2}\left\|\varepsilon^{\mathfrak{n}}\right\|_{2} \leqslant \sqrt{\lambda_{d+1}}, \quad n=1,2, \ldots, l,
$$

where $\varepsilon_{n}(n=1,2, \cdots, l)$ are the unit vectors with $n$-th element being 1 . Hence, the POD basis $\Phi_{\mathfrak{u}}=$ $\left(\phi_{1}, \phi_{2}, \cdots, \phi_{d}\right)$ is optimal. 
Remark 3.1. Because the order $M-1$ of the matrix $A_{\mathfrak{u}} A_{\mathfrak{u}}^{\top}$ is far larger than the order $l$ of the matrix $A_{\mathfrak{u}}^{\top} A_{\mathfrak{u}}$, i.e., the number of the spatial internal nodes $M-1$ is far larger than that of the snapshots $l$, but both positive eigenvalues $\lambda_{i}(i=1,2, \ldots, r)$ are identical. As a consequence, we may first seek the eigenvalues $\lambda_{i}$ and the eigenvectors $\psi_{\mathfrak{i}}(\mathfrak{i}=1,2, \ldots, r)$ of $\boldsymbol{A}_{\mathfrak{u}}^{\top} \boldsymbol{A}_{\mathfrak{u}}$, we then compute out the eigenvectors $\varphi_{i}$ of $A_{\mathfrak{u}} A_{u}^{\top}$ via the formula $\varphi_{i}=A_{\mathfrak{u}} \psi_{i} / \sqrt{\lambda_{i}}(i=1,2, \ldots, r)$ such that the formulation of POD basis becomes simpler.

\subsection{Establishment of the ROEFD scheme for the Riesz space FOCNDE}

From Section 3.1, we can obtain the initial $l(l \leqslant N)$ FOECNFD solutions $\mathbf{u}_{d}^{n}=\Phi_{u} \Phi_{u}^{\top} \mathbf{u}^{n}=$ : $\Phi_{u} \beta_{d}^{n}(n=1,2, \ldots, L)$, where $u_{d}^{n}=\left(u_{d, 1}^{n}, u_{d, 2}^{n}, \cdots, u_{d, M-2}^{n}, u_{d, M-1}^{n}\right)^{\top}$ and $\beta_{d}^{n}=\left(\beta_{1}^{n}, \beta_{2}^{n}, \ldots, \beta_{d}^{n}\right)^{\top}$. Now, by replacing $\mathbf{U}^{n}$ in (2.3) with $\mathbf{U}_{d}^{n}=\Phi_{u} \boldsymbol{\beta}_{d}^{n}(n=l+1, l+2, \ldots, N)$, we can attain the ROECNFD scheme as follows:

$$
\left\{\begin{aligned}
\Phi \beta_{d}^{n}= & \Phi_{u} \Phi_{u}^{\top} \mathbf{u}^{n}, n=1,2, \ldots, l, \\
\Phi_{u} \beta_{d}^{n}= & \Phi_{u} \beta_{d}^{n-1}+\frac{1}{2} \mathbf{D}\left(2 \Phi_{u} \beta_{d}^{n-1}+D \Phi_{u} \beta_{d}^{n-1}+\tau F\left(\Phi_{u} \beta_{d}^{n-1}\right)\right) \\
& +\frac{\tau}{2}\left[F\left(\Phi_{u} \beta_{d}^{n-1}\right)+F\left(\Phi_{u} \beta_{d}^{n-1}+D \Phi_{u} \beta_{d}^{n-1}+\tau F\left(\Phi_{u} \beta_{d}^{n-1}\right)\right)\right], n=l+1, l+2, \ldots, N, \\
u_{d}^{n}= & \Phi \beta_{d}^{n}, \quad n=1,2, \ldots, N,
\end{aligned}\right.
$$

where $\mathbf{U}^{\mathrm{n}}(\mathrm{n}=1,2, \ldots, \mathrm{l})$ are the known classical CNFD solution vectors for the classical CNFD scheme (2.3). The ROECNFD scheme (3.2) is simplified into the following:

$$
\left\{\begin{aligned}
\beta_{d}^{n}= & \Phi_{u}^{\top} \mathbf{u}^{n}, 1 \leqslant n \leqslant l, \\
\beta_{d}^{n}= & \beta_{d}^{n-1}+\frac{1}{2} \Phi_{u}^{\top} \mathbf{D}\left[2 \Phi_{u} \beta_{d}^{n-1}+D \Phi_{u} \beta_{d}^{n-1}+\tau F\left(\Phi_{u} \beta_{d}^{n-1}\right)\right] \\
& +\frac{\tau}{2} \Phi_{u}^{\top}\left[F\left(\Phi_{u} \beta_{d}^{n-1}\right)+F\left(\Phi_{u} \beta_{d}^{n-1}+D \Phi_{u} \beta_{d}^{n-1}+\tau F\left(\Phi_{u} \beta_{d}^{n-1}\right)\right)\right], \quad l+1 \leqslant n \leqslant N, \\
u_{d}^{n}= & \Phi_{u} \beta_{d}^{n}, 1 \leqslant n \leqslant N .
\end{aligned}\right.
$$

Remark 3.2. Because the classical CNFD scheme (2.3) contains $(M-1)$ unknowns at each time node, whereas the ROECNFD scheme (3.3) at the same time node only has $d$ unknowns $(d \ll M-1)$, the ROECNFD scheme (3.3) is superior to the classical CNFD scheme (2.3).

\section{The existence, stability, and convergence of the ROECNFD solutions and the flowchart for solving the ROECNFD scheme}

\subsection{The existence, stability, and convergence of the ROECNFD solutions}

The result of the existence, stability, and convergence of the ROECNFD solutions are stated as follows.

Theorem 4.1. Under the conditions of Theorem 2.2, the ROECNFD scheme (3.3) has a unique set of solutions $\left\{\mathbf{U}_{\mathrm{d}}^{\mathfrak{n}}\right\}_{\mathfrak{n}=1}^{\mathrm{N}}$, which is stable and convergent and has the following error estimates

$$
\max _{1 \leqslant i \leqslant n}\left\|\mathbf{U}^{i}-\mathbf{U}_{d}^{i}\right\|_{\infty} \leqslant E(n) \sqrt{\lambda_{d+1}}, \quad n=1,2, \ldots, N,
$$

where $\mathrm{U}^{i}(i=1,2, \ldots, N)$ is the series of the CNFD solutions for the classical CNFD scheme (2.3), $E(n)=1$ $(0 \leqslant n \leqslant l)$, and $E(n)=\left[1+\tau\left(\beta_{1}+\beta_{2}\right)\left(2+\beta_{1} \tau+\|D\|_{\infty}\right) / 2\right]^{n-l}(l+1 \leqslant n \leqslant N)$. Moreover, the errors between the analytic solution vectors $\tilde{\mathbf{u}}\left(t_{n}\right)=\left[u\left(t_{n}, x_{1}\right), u\left(t_{n}, x_{2}\right), \ldots, u\left(t_{n}, x_{M-1}\right)\right]^{\top}(n=1,2, \ldots, N)$ for the Riesz space FODEs (1.1) and the ROECND solutions $\mathbf{u}_{\mathrm{d}}^{\mathrm{n}}$ for the ROECNFD scheme (3.3) have the following estimates

$$
\max _{1 \leqslant i \leqslant n}\left\|\tilde{\mathbf{U}}^{i}-\mathbf{U}_{d}^{i}\right\|_{\infty} \leqslant Q\left[\tau^{2}+h^{2}+E(n) \sqrt{\lambda_{d+1}}\right], \quad n=1,2, \ldots, N .
$$


Proof. By using $\mathbf{U}_{\mathrm{d}}^{\mathrm{n}}=\Phi \beta_{\mathrm{d}}^{n}(\mathrm{n}=1,2, \ldots, \mathrm{N})$, the ROECNFD scheme (3.2) is restored into the following:

$$
\begin{aligned}
\mathbf{U}_{\mathrm{d}}^{n}= & \boldsymbol{\Phi}_{\mathfrak{u}} \boldsymbol{\Phi}_{\mathfrak{u}}^{\top} \mathbf{U}^{\mathrm{n}}, \quad 1 \leqslant \mathrm{n} \leqslant \mathrm{l}, \\
\mathbf{U}_{\mathrm{d}}^{\mathrm{n}}= & \mathbf{U}_{\mathrm{d}}^{\mathrm{n}-1}+\frac{1}{2} \mathbf{D}\left(2 \mathbf{U}_{\mathrm{d}}^{\mathrm{n}-1}+\mathbf{D U}_{\mathrm{d}}^{\mathrm{n}-1}+\tau \mathrm{F}\left(\mathbf{U}_{\mathrm{d}}^{\mathrm{n}-1}\right)\right) \\
& +\frac{\tau}{2}\left[\mathbf{F}\left(\mathbf{U}_{\mathrm{d}}^{\mathrm{n}-1}\right)+\mathbf{F}\left(\mathbf{U}_{\mathrm{d}}^{\mathrm{n}-1}+\mathbf{D U}_{\mathrm{d}}^{\mathrm{n}-1}+\tau \mathbf{F}\left(\mathbf{U}_{\mathrm{d}}^{\mathrm{n}-1}\right)\right)\right], \quad l+1 \leqslant \mathrm{n} \leqslant \mathrm{N} .
\end{aligned}
$$

Because the classical CNFD solutions $\mathbf{U}^{n}(n=1,2, \ldots, l)$ are known and stable, from (4.3), we attain a unique series of solutions $\mathbf{U}_{d}^{n}=\Phi_{\mathfrak{u}} \boldsymbol{\Phi}_{\mathfrak{u}}^{\top} \mathbf{U}^{n}(n=1,2, \ldots, l)$, which are stable since $\left\|\mathbf{U}_{d}^{n}\right\|_{2} \leqslant\left\|\mathbf{U}^{n}\right\|_{2}$. Furthermore, when the $\mathrm{U}_{\mathrm{d}}^{\mathrm{n}-1}$ in the right hand side of (4.4) are known, from (4.4), we can compute out $\mathrm{u}_{\mathrm{d}}^{\mathrm{n}-1}(\mathrm{n}=\mathrm{l}+1, l+2, \ldots, \mathrm{N})$. Therefore, the ROECNFD scheme (4.4) exists a unique set of solutions $\left\{\mathbf{U}_{d}^{n}\right\}_{\mathfrak{n}=l+1}^{N}$. Because (4.4) is the same form as (2.3), by using the same techniques as proving Theorem 2.2, we can demonstrate that the ROECNFD solutions $\left\{\mathbf{U}_{d}^{n}\right\}_{n=l+1}^{N}$ are stable. Thus, the ROECNFD scheme (3.3) has a unique set of stable solutions $\left\{\mathbf{U}_{d}^{n}\right\}_{\mathfrak{n}=1}^{N}$. Furthermore, by using Lax's stability theorem (see e.g., $[4,36])$, we attain that the ROECNFD solutions $\left\{\mathbf{u}_{d}^{n}\right\}_{\mathfrak{n}=1}^{N}$ are convergent.

When $n=1,2, \cdots, l$, from the properties of norm and (3.1), we immediately obtain the following error estimates

$$
\left\|\mathbf{U}^{i}-\mathbf{U}_{\mathrm{d}}^{\mathrm{i}}\right\|_{\infty} \leqslant\left\|\mathbf{U}^{\mathrm{i}}-\mathbf{U}_{\mathrm{d}}^{\mathrm{i}}\right\|_{2}=\left\|\mathbf{U}^{\mathrm{i}}-\boldsymbol{\Phi}_{\mathbf{u}} \boldsymbol{\Phi}_{\mathbf{u}}^{\top} \mathbf{U}^{\mathrm{i}}\right\|_{2} \leqslant \sqrt{\lambda_{\mathrm{d}+1}}, \quad 1 \leqslant i \leqslant n \leqslant l .
$$

Thus, we have

$$
\max _{1 \leqslant i \leqslant n}\left\|\mathbf{U}^{i}-\mathbf{u}_{\mathrm{d}}^{i}\right\|_{\infty} \leqslant \sqrt{\lambda_{\mathrm{d}+1}}, \quad 1 \leqslant \mathrm{n} \leqslant \mathrm{l}
$$

Let $\boldsymbol{e}_{\mathrm{n}}=\mathbf{U}^{\mathrm{n}}-\mathbf{u}_{\mathrm{d}}^{\mathrm{n}}$. By subtracting (4.4) from (2.3), we gain

$$
\begin{aligned}
& \mathbf{e}_{n}=e_{n-1}+\frac{1}{2} \mathbf{D}\left(2 e_{n-1}+D e_{n-1}+\tau F\left(\mathbf{U}^{n-1}\right)-\tau F\left(\mathbf{U}_{d}^{n-1}\right)\right)+\frac{\tau}{2}\left[F\left(\mathbf{U}^{n-1}\right)\right. \\
& \left.+\mathbf{F}\left(\mathbf{U}^{n-1}+\mathbf{D U}^{\mathrm{n}-1}+\tau \mathbf{F}\left(\mathbf{U}^{\mathrm{n}-1}\right)\right)-\mathbf{F}\left(\mathbf{U}_{\mathrm{d}}^{\mathrm{n}-1}\right)-\mathbf{F}\left(\mathbf{U}_{\mathrm{d}}^{\mathrm{n}-1}+\mathbf{D U}_{\mathrm{d}}^{\mathrm{n}-1}+\tau \mathrm{F}\left(\mathbf{U}_{\mathrm{d}}^{\mathrm{n}-1}\right)\right)\right] \\
& =\frac{\tau}{2}\left[\mathbf{F}\left(\mathbf{U}^{n-1}+\mathbf{D U}^{n-1}+\tau \mathbf{F}\left(\mathbf{U}^{n-1}\right)\right)-\mathbf{F}\left(\mathbf{U}_{\mathrm{d}}^{n-1}+\mathbf{D U}_{\mathrm{d}}^{n-1}+\tau \mathbf{F}\left(\mathbf{U}_{\mathrm{d}}^{n-1}\right)\right)\right] \\
& +\left(\mathbf{I}+\mathbf{D}+\frac{1}{2} \mathbf{D}^{2}\right) \mathbf{e}_{\mathrm{n}-1}+\frac{\tau}{2}(\mathbf{I}+\mathbf{D})\left[\mathbf{F}\left(\mathbf{U}^{\mathrm{n}-1}\right)-\mathbf{F}\left(\mathbf{U}_{\mathrm{d}}^{\mathrm{n}-1}\right)\right], \quad l+1 \leqslant \mathrm{n} \leqslant \mathrm{N} .
\end{aligned}
$$

When $\|\mathbf{I}+\mathbf{D}\|_{\infty} \leqslant 1$, we have

$$
\left\|\mathbf{I}+\mathbf{D}+\frac{1}{2} \mathbf{D}^{2}\right\|_{\infty}=\left\|\frac{1}{2}\left[\mathbf{I}+(\mathbf{I}+\mathbf{D})^{2}\right]\right\|_{\infty} \leqslant \frac{1}{2}\left(1+\|\mathbf{I}+\mathbf{D}\|_{\infty}\right) \leqslant 1 .
$$

Thus, by (1.2), from (4.6), we have

$$
\begin{aligned}
\left\|\boldsymbol{e}_{n}\right\|_{\infty} \leqslant & \left\|\mathbf{I}+\mathbf{D}+\frac{1}{2} \mathbf{D}^{2}\right\|_{\infty}\left\|\boldsymbol{e}_{n-1}\right\|_{\infty}+\frac{\tau}{2}\|\mathbf{D}\|_{\infty}\left(\beta_{1}\left\|\boldsymbol{e}_{n-1}\right\|_{\infty}+\beta_{2}\left\|\boldsymbol{e}_{n-m}\right\|_{\infty}\right) \\
& +\frac{\tau}{2}\left[\left(\beta_{1}\left\|\boldsymbol{e}_{n-1}\right\|_{\infty}+\beta_{2}\left\|\boldsymbol{e}_{n-m}\right\|_{\infty}\right)+\beta_{1}\left(\|\mathbf{I}+\mathbf{D}\|_{\infty}\left\|\boldsymbol{e}_{n-1}\right\|_{\infty}\right.\right. \\
& \left.+\tau\left(\beta_{1}\left\|\boldsymbol{e}_{n-1}\right\|_{\infty}+\beta_{2}\left\|\boldsymbol{e}_{n-m}\right\|_{\infty}\right)+\beta_{2}\left\|\mathbf{e}_{n-m}\right\|_{\infty}\right], \quad n=l+1, l+2, \ldots, N .
\end{aligned}
$$

Set $\Upsilon=\left(\beta_{1}+\beta_{2}\right)\left(2+\beta_{1} \tau+\|\mathbf{D}\|_{\infty}\right) / 2$ and $\Lambda_{n}=\max _{1 \leqslant i \leqslant n}\left\|\boldsymbol{e}_{i}\right\|_{\infty}=\max _{1 \leqslant i \leqslant n}\left\|\mathbf{U}^{i}-\mathbf{U}_{d}^{i}\right\|_{\infty}$. By (4.7) and $\|\mathbf{I}+\mathbf{D}\|_{\infty} \leqslant 1$, from (4.8), we obtain

$$
\begin{aligned}
\Lambda_{n} \leqslant & \Lambda_{n-1}+\frac{\tau}{2}\|D\|_{\infty}\left(\beta_{1} \Lambda_{n-1}+\beta_{2} \Lambda_{n-1}\right) \\
& +\frac{\tau}{2}\left[\left(\beta_{1} \Lambda_{n-1}+\beta_{2} \Lambda_{n-1}\right)+\beta_{1}\left(\Lambda_{n-1}+\tau\left(\beta_{1} \Lambda_{n-1}+\beta_{2} \Lambda_{n-1}\right)+\beta_{2} \Lambda_{n-1}\right]\right. \\
\leqslant & \left(1+\Upsilon_{\tau}\right) \Lambda_{n-1}, \quad n=l+1, l+2, \ldots, N .
\end{aligned}
$$


From (4.9) and (4.5), we have

$$
\max _{1 \leqslant i \leqslant n}\left\|\mathbf{U}^{i}-\mathbf{U}_{d}^{i}\right\|_{\infty} \leqslant(1+\Upsilon \tau)^{n-l} \Lambda_{l} \leqslant E(n) \sqrt{\lambda_{d+1}}, \quad n=l+1, l+2, \ldots, N .
$$

Combining (4.5) with (4.10) yields (4.1) and combining Theorem 2.2 with (4.1) yields (4.2). This accomplishes the demonstration of Theorem 4.1.

Remark 4.2. The error factors $\sqrt{\lambda_{d+1}}$ and $E(n)=\left[1+\tau\left(\beta_{1}+\beta_{2}\right)\left(2+\beta_{1} \tau+\|\mathbf{D}\|_{\infty}\right) / 2\right]^{n-l}(l+1 \leqslant n \leqslant$ $\mathrm{N}$ ) in Theorem 4.1 are caused by the order-reduction for the classical CNFD scheme (2.3) and by the extrapolating iteration, respectively. They can, respectively, serve as the suggestions of choice of the number $d$ of POD bases and update of the POD basis in the actual numerical computations. Though the ROECNFD solutions lose some accuracy contrasting the classical CNFD ones, they can greatly save degrees of freedom so as to lessen the truncated error amassing and improve computational efficiency, as shown in the numerical experiments in Section 5.

Remark 4.3. When $\|\mathbf{I}+\mathbf{D}\|_{\infty} \leqslant 1$, we have $\|\mathbf{D}\|_{\infty}=\|\mathbf{I}+\mathbf{D}-\mathbf{I}\|_{\infty} \leqslant\|\mathbf{I}+\mathbf{D}\|_{\infty}+\|\mathbf{I}\|_{\infty} \leqslant 2$. Therefore, $\Upsilon=\left(\beta_{1}+\beta_{2}\right)\left(2+\beta_{1} \tau+\|\mathbf{D}\|_{\infty}\right) / 2 \leqslant\left(\beta_{1}+\beta_{2}\right)\left(2+0.5 \beta_{1} \tau\right)$ is a finite real-number such that $\Upsilon \tau$ is usually quite small. In addition, a lot of numerical experiments have shown that the eigenvalues $\lambda_{j}(j=1,2, \ldots, L)$ of the matrix $A_{u} A_{u}^{\top}$ are usually decreasing quickly to be close to zero. Therefore, if only we choose $d$ such that $\left[1+\tau\left(\beta_{1}+\beta_{2}\right)\left(2+\beta_{1} \tau+\|\mathbf{D}\|_{\infty}\right) / 2\right]^{N-l} \sqrt{\lambda_{d+1}} \leqslant \min \left\{\tau^{2}, h^{2}\right\}$, we can ensure that the ROECNFD solutions attain the optimal order convergence.

\subsection{Flowchart of solving ROECNFD scheme}

In the following, we provide the flowchart for solving the ROESTCFE scheme of the Riesz space FODEs with a nonlinear source function and delay on a bounded domain, which consists of the following six steps.

Step 1. Extract the snapshots $u^{i}(i=1,2, \ldots, l)$ from the classical CNFD solutions for the following classical CNFD scheme (at the first $l$ steps, usually $l=20$ ):

$$
\begin{aligned}
\mathbf{U}^{n}= & \mathbf{U}^{n-1}+\frac{1}{2} \mathbf{D}\left(2 \mathbf{U}^{n-1}+\mathbf{D} \mathbf{U}^{n-1}+\tau \mathbf{F}\left(\mathbf{U}^{n-1}\right)\right) \\
& +\frac{\tau}{2}\left[\mathbf{F}\left(\mathbf{U}^{n-1}\right)+\mathbf{F}\left(\mathbf{U}^{n-1}+\mathbf{D U}^{n-1}+\tau \mathbf{F}\left(\mathbf{U}^{n-1}\right)\right)\right], n=1,2, \ldots, l,
\end{aligned}
$$

subject to the initial condition

$$
\mathbf{u}^{0}=\left[u_{1}^{0}, u_{2}^{0}, \ldots, u_{M-2}^{0}, u_{M-1}^{0}\right]^{\top}, u_{i}^{0}=\varphi(0, i h), u_{i}^{k}=\varphi(k \tau, i h), i=1,2, \ldots, M-1, k<0 .
$$

Step 2. Assemble the snapshot matrix $\mathbf{A}_{\mathfrak{u}}=\left[\mathbf{u}^{1}, \mathbf{u}^{2}, \ldots, \mathbf{U}^{\mathbf{l}}\right]$.

Step 3. Compute the eigenvalues $\lambda_{1} \geqslant \lambda_{2} \geqslant \cdots \geqslant \lambda_{r}>0$ and the corresponding eigenvectors $\psi^{j}(j=$ $1,2, \ldots, r)$ for the matrix $\boldsymbol{A}_{\mathfrak{u}}^{\top} \boldsymbol{A}_{\mathfrak{u}}$, where $r=\operatorname{dim}\left\{\mathbf{U}^{1}, \mathbf{U}^{2}, \ldots, \mathbf{U}^{\mathrm{l}}\right\}$.

Step 4. For the spatial step-size $h$, the time step-size $\tau$, and the desired error $\delta=O\left(\tau^{2}, h^{2}\right)$, determine the number $\mathrm{d}$ of POD basis such that $\lambda_{\mathrm{d}+1} \leqslant \delta^{2}$ is satisfied.

Step 5. Produce the POD basis $\Phi=\left[\varphi_{1}, \varphi_{2}, \ldots, \varphi_{d}\right]$ by $\varphi_{i}=A_{u} \psi_{i} / \sqrt{\lambda_{i}}(i=1,2, \ldots, d)$ and attain the ROECNFD solutions by solving the following ROECNFD scheme:

$$
\left\{\begin{aligned}
\beta_{d}^{n}= & \Phi_{u}^{\top} \mathbf{U}^{n}, \quad 1 \leqslant n \leqslant l, \\
\boldsymbol{\beta}_{d}^{n}= & \beta_{d}^{n-1}+\frac{1}{2} \Phi_{u}^{\top} \mathbf{D}\left(2 \Phi_{u} \beta_{d}^{n-1}+D \Phi_{u} \beta_{d}^{n-1}+\tau F\left(\Phi_{u} \beta_{d}^{n-1}\right)\right) \\
& +\frac{\tau}{2} \Phi_{u}^{\top}\left[F\left(\Phi_{u} \beta_{d}^{n-1}\right)+F\left(\Phi_{u} \beta_{d}^{n-1}+D \Phi_{u} \beta_{d}^{n-1}+\tau F\left(\Phi_{u} \beta_{d}^{n-1}\right)\right)\right], l+1 \leqslant n \leqslant N, \\
u_{d}^{n}= & \Phi_{u} \beta_{d}^{n}, 1 \leqslant n \leqslant N,
\end{aligned}\right.
$$

which only includes d unknowns.

Step 6. If $\left.\left.\| \mathbf{U}_{d}^{n}-\mathbf{U}_{d}^{n+1}\right)\left\|_{\infty} \leqslant\right\| \mathbf{u}_{d}^{n-1}-\mathbf{U}_{d}^{n}\right) \|_{\infty}(n=l, l+1, \ldots, N-1)$, end. Else, let $\mathbf{U}^{i}=\mathbf{u}_{d}^{n-l-i}(i=$ $1,2, \ldots, l)$ and return to Step 2. 


\section{Numerical experiments}

In this section, we give some numerical experiments to illustrate the superiority of the ROECNFD scheme (3.3) for the Riesz space FODEs.

In the Riesz space FODEs (1.1) with a nonlinear source function and delay, we take $0 \leqslant \mathrm{t} \leqslant 750$, i.e., $T=750,0 \leqslant x \leqslant 16000$, i.e., $\mathrm{L}=16000, \mathrm{~K}=1, \alpha=1.5, \mathrm{~s}=0.01$, the boundary value function $g(t)=0.22$, the nonlinear source term $f(t, x, u(t, x), u(t-0.01))=u(t, x) u(t-0.01, x)$, and the initial function $\left.\varphi(t, x)=0.22+0.05\left\{4[\cosh (100 x-600000)]^{-2}-\cosh (0.0025 x-16.25)\right]^{-2}\right\}$. In this case, it is very difficult to find the analytical solution for the Riesz space FODEs so that we can only find their numerical solutions.

Let $\tau=h=0.01$. We first compute the initial $l=20$ solution vectors $U^{n}(n=1,2, \ldots, 20)$ by the classical CNFD scheme (2.3) as the snapshots to form snapshot matrix $\mathbf{A}_{\mathfrak{u}}=\left[\mathbf{U}^{1}, \mathbf{U}^{2}, \ldots, \mathbf{U}^{20}\right]$. Then we find the eigenvalues $\lambda_{1} \geqslant \lambda_{2} \geqslant \cdots \geqslant \lambda_{20} \geqslant 0$ and the corresponding eigenvectors $\varphi_{j}(j=1,2, \ldots, 20)$ according to Steps 3 and 5 in Subsection 4.2. By reckoning, we attain that $\sqrt{\lambda_{7}} \leqslant 3.5 \times 10^{-4}$. Therefore, we take the POD basis $\Phi=\left[\boldsymbol{\varphi}_{1}, \boldsymbol{\varphi}_{2}, \ldots, \boldsymbol{\varphi}_{6}\right]$ and find the ROECNFD solutions $u_{\mathrm{di}}^{\mathrm{n}}(\mathrm{n}=1,2, \ldots, 75000$ and $i=1,2, \ldots, 1600000$, i.e., $0<t \leqslant 750$ and $0 \leqslant x \leqslant 16000)$ by means of the ROECNFD scheme (3.3), depict graphically them in Figure 1. In order to compare with the ROECNFD solutions, we also compute out the classical CNFD solutions $u_{i}^{n}(n=1,2, \ldots, 75000$ and $i=1,2, \ldots, 1600000$, i.e., $0<t \leqslant 750$ and $0 \leqslant x \leqslant 16000$ ) by means of the classical CNFD scheme (2.3), depict graphically them in Figure 2 . Even if Figure 2 and Figure 1 are almost same, by checking carefully, we found that the results of the ROECNFD solutions in Figure 1 are better than those of the classical CNFD solutions in Figure 2. Because the classical CNFD scheme at each time node has one million and six hundred thousand unknowns, whereas the ROECNFD scheme at the same time node only has six unknowns, the ROECNFD scheme can greatly retard the truncated error amassing in the calculating process and improves the accuracy for the ROECNFD numerical solutions. By operating records from solving the classical CNFD scheme and the ROECNFD scheme in the same Laptop (Microsoft Surface Book: Int Core i7 Processor, 16GB RAM), we find that the CPU time-consuming for solving the classical CNFD scheme on $0 \leqslant t \leqslant 750$ is seven hundred and sixty-two minutes, but the CPU time-consuming for solving the ROECNFD scheme is less than six minutes, that is that the CPU time-consuming for solving the classical CNFD scheme is one hundred and twenty-six times more than that for solving the ROECNFD scheme. This shows that the ROECNFD scheme is far superior to the classical CNFD scheme.

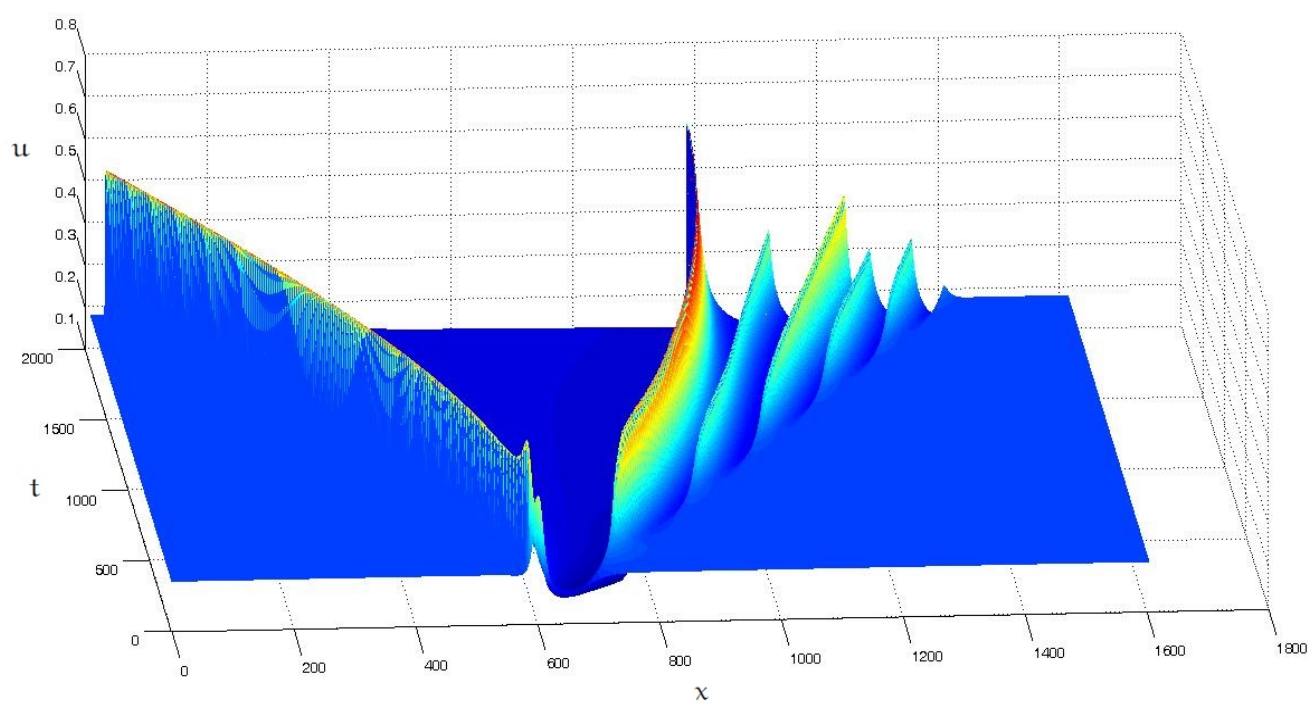

Figure 1: The classical CNFD solutions when $0 \leqslant t \leqslant 750$ and $0 \leqslant x \leqslant 16000$. 


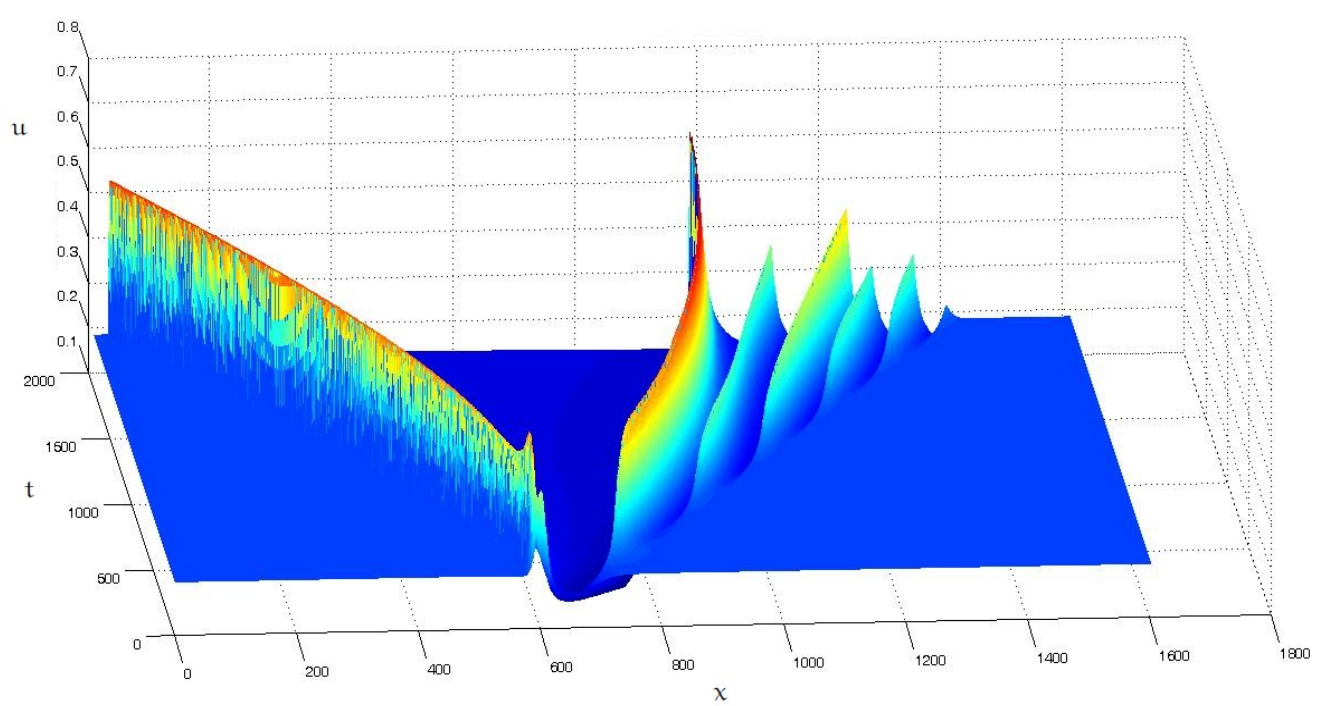

Figure 2: The ROENBE solutions when $0 \leqslant t \leqslant 750$ and $0 \leqslant x \leqslant 16000$.

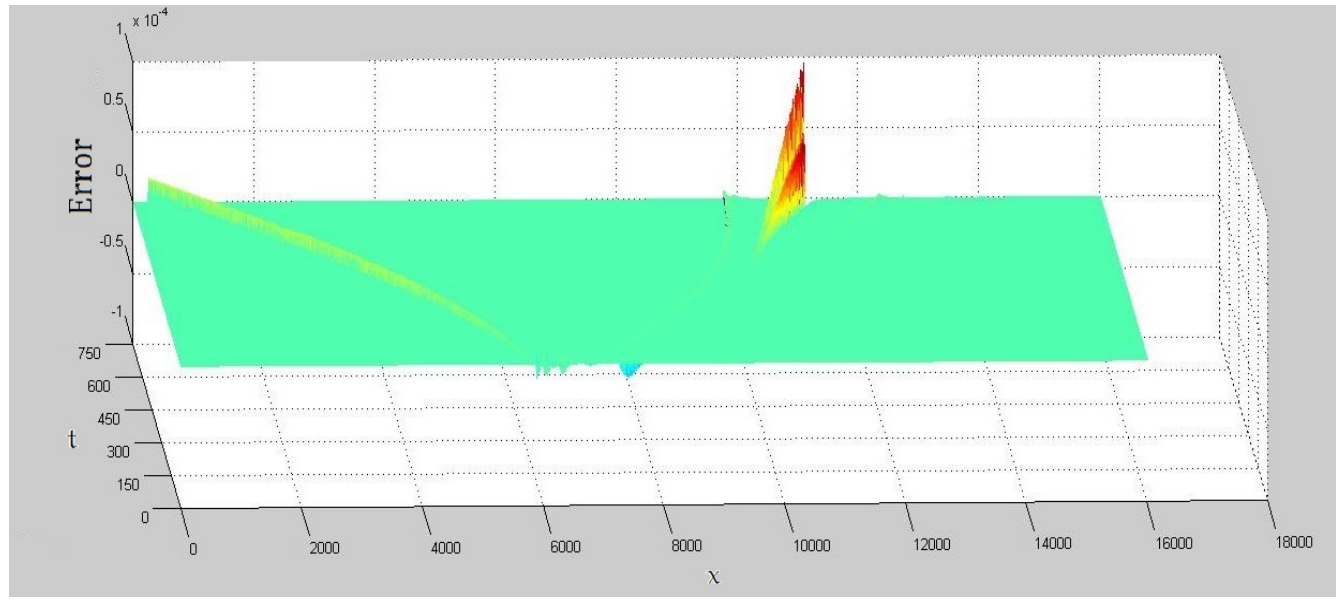

Figure 3: The error photo between the classical CNFD solutions and the ROECNFD solutions on $0 \leqslant t \leqslant 750$.

Figure 3 shows the error photo between the classical CNFD solutions and the ROECNFD solutions on $0 \leqslant t \leqslant 750$, which is consistent with the theoretical result of (4.1) in the Theorem 4.1, because both theoretical and numerical errors are $\mathrm{O}\left(10^{-4}\right)$ when $\tau=h=0.01$. This implies that the ROECNFD scheme is efficient and feasible for solving the Riesz space FODEs (1.1).

\section{Conclusions and discussion}

In this article, we have built the ROECNFD scheme containing very few unknowns but possessing fully second-order accuracy for the Riesz space FODEs with a nonlinear source function and delay on a bounded domain, analyzed the the existence, stability, and convergence of the ROECNFD solutions. we have also used some numerical experiments to check the feasibility and effectiveness of the ROECNFD scheme and to verity that the numerical computing consequences are coincided with the theoretical analysis ones. Especially, the ROECNFD scheme has more advantageous than the classical CNFD scheme, because the ROECNFD scheme can greatly save more CPU time-consuming and degrees of freedom than the classical CNFD scheme. Moreover, because the ROECNFD scheme for the Riesz space FODEs with a nonlinear source function and delay is first presented, it is improvement over the existing other 
reduced-order methods as mentioned in Section 1.

Even if we only research the order-reduction for the classical CNFD scheme of the Riesz space FODEs with a nonlinear source function and delay on a bounded domain, because the classical CNFD scheme can solve the Riesz space FODEs in the two and three-dimensional unbounded domain (see, e.g., [17]), the ROECNFD scheme can be easily and effectively used to reduce the order for the Riesz space FODEs in the two and three-dimensional domain.

\section{Acknowledgment}

This work is jointly supported by the National Science Foundation of China (Grant Nos. 11671106 and 11461026).

\section{References}

[1] P. Benner, A. Cohen, M. Ohlberger, K. Willcox, Model Reduction and Approximation: Theory and Algorithm, Computational Science \& Engineering, SIAM, (2017). 1

[2] W. Cazemier, R. W. C. P. Verstappen, A. E. P. Veldman, Proper orthogonal decomposition and low-dimensinal models for driven cavity flows, Physics of Fluids, 10 (1998), 1685-1699. 1

[3] H. Chou, B. Lee, C. Chen, The transient infiltration process for seepage flow from cracks, Advances in Subsurface Flow and Transport: Eastern and Western Approaches III, (2006). 1

[4] T. J. Chung, Computational Fluid Dynamics, Cambridge University Press, Cambridge, (2002). 4.1

[5] K. Fukunaga, Introduction to Statistical Recognition, Academic Press, Boston, (1990). 1

[6] J. S. Hesthaven, G. Rozza, B. Stamm, Certified Reduced Basis Methods for Parametrized Partial Differential Equations, BCAM SpringerBriefs, Springer, Cham; BCAM Basque Center for Applied Mathematics, Bilbao, (2016). 1

[7] P. Holmes, J. L. Lumley, G. Berkooz, Turbulence, Coherent Structures, Dynamical Systems and Symmetry, Cambridge University Press, Cambridge, (1996). 1

[8] A. X. Huang, A new decomposition for solving percolation equations in porous media, Third International Symposium, on Aerothermodynamics of Internal Flows, Beijing, China, 1 (1996), 417-420. 1

[9] I. T. Jolliffe, Principal Component Analysis, (Second edition) Springer Series in Statistics, Springer-Verlag, New York, (2002). 1

[10] K. Kunisch, S. Volkwein, Galerkin proper orthogonal decomposition methods for parabolic problems, Numer. Math., 90 (2001), 117-148. 1

[11] K. Kunisch, S. Volkwein, Galerkin proper orthogonal decomposition methods for a general equation in fluid dynamischs, SIAM J. Numer. Anal., 40 (2002), 492-515. 1

[12] D. Kumar, J. Singh, D. Baleanu, Numerical Computation of a Fractional Model of Differential-Difference Equation, J. Comput. Nonlinear Dynam., 11 (2016), 6 pages. 1

[13] D. Kumar, J. Singh, D. Baleanu, A fractional model of convective radial fins with temperature-dependent thermal conductivity, Rom. Rep. Phys., 69 (2017), 13 Pages. 1

[14] D. Kumar, J. Singh, D. Baleanu, A hybrid computational approach for Klein-Gordon equations on Cantor sets, Nonlinear Dynam., 87 (2017), 511-517. 1

[15] D. Kumar, J. Singh, D. Baleanu, Modified Kawahara equation within a fractional derivative with non-singular kernel, Therm. Sci., 2017 (2017), 10 Pages. 1

[16] D. Kumar, J. Singh, S. Kumar, B. P. Singh, Numerical computation of nonlinear shock wave equation of fractional order, Ain Shams Eng. J., 6 (2015), 605-611. 1

[17] F. Liu, P. Zhuang, Q. X. Liu, Numerical methods of fractional partial differential equations and their applications, Science Press (In Chinease), (2015). 1, 2, 6

[18] Z. Luo, J. Chen, I. M. Navon, X. Yang, Mixed finite element formulation and error estimates based on proper orthogonal decomposition for the non-stationary Navier-Stokes equations, SIAM J. Numer. Anal., 47 (2008/09), 1-19. 1

[19] Z. Luo, J. Q. Gao, A POD-based reduced-order finite difference time-domain extrapolating scheme for the 2D Maxwell equations in a lossy medium, J. Math. Anal. Appl., 444 (2016), 433-451. 1

[20] Z. Luo, H. Li, P. Sun, J. Gao, A reduced-order finite difference extrapolation algorithm based on POD technique for the non-stationary Navier-Stokes equations, Appl. Math. Model., 37 (2013), 5464-5473. 1

[21] Z. Luo, H. Li, Y. Zhou, X. Huang, A reduced FVE formulation based on POD method and error analysis for twodimensional viscoelastic problem, J. Math. Anal. Appl., 385 (2012), 310-321. 1

[22] Z. Luo, H. Li, Y. Zhou, Z. Xie, A reduced finite element formulation based on POD method for two-dimensional solute transport problems, J. Math. Anal. Appl., 385 (2012), 371-383. 1

[23] Z. Luo, Z. Xie, Y. Shang, J. Chen, A reduced finite volume element formulation and numerical simulations based on POD for parabolic problems, J. Comput. Appl. Math., 235 (2011), 2098-2111. 1 
[24] Z. Luo, X. Yang, Y. Zhou, A reduced finite difference scheme based on singular value decomposition and proper orthogonal decomposition for Burgers equation, J. Comput. Appl. Math., 229 (2009), 97-107. 1, 3.1

[25] H. V. Ly, H. T. Tran, Proper orthogonal decomposition for flow calculations and optimal control in a horizontal CVD reactor, Quart. Appl. Math., 60 (1989), 631-656. 1

[26] M. M. Meerschaert, C. Tadjeran, Finite difference approximations for two-sided space-fractional partial differential equations, Appl. Numer. Math., 56 (2006), 80-90. 1

[27] N. Petford, M. A. Koenders, Seepage flow and consolidation in a deforming porous medium, EGS-AGU-EUG Joint Assembly, (2003). 1

[28] A. Quarteroni, A. Manzoni, F. Negri, Reduced Basis Methods for Partial Differential Equations, Springer, Cham, (2016). 1

[29] F. M. Selten, Baroclinic empirical orthogonal functions as basis functions in an atmospheric model, J. Atmos. Sci., 54 (1997), 2099-2114. 1

[30] L. Sirovich, Turbulence and the dynamics of coherent structures: part I-III, Quart. Appl. Math., 45 (1987), 561-590. 1

[31] P. Sun, Z. Luo, Y. Zhou, Some reduced finite difference schemes based on a proper orthogonal decomposition technique for parabolic equations, Appl. Numer. Math., 60 (2010), 154-164. 1, 3.1

[32] N. I. Thusyanthan, S. P. G. Madabhushi, Scaling of seepage flow velocity in centrifuge models, Cambridge University Engineering Department Technical Report CUED/D-SOILS/TR326, 2003 (2003), 13 pages. 1

[33] H. Xia, Z. Luo, An optimized finite difference iterative scheme based on POD technique for the 2D viscoelastic wave equation, Appl. Math. Mech., 38 (2017), 1721-1732. 1

[34] H. Xia, Z. Luo, A POD-based optimized finite difference $C N$ extrapolated implicit scheme for the $2 D$ viscoelastic wave equation, Math. Methods Appl. Sci., 40 (2017), 6880-6890. 1

[35] S. P. Yang, Finite difference method for Riesz space fractional diffusion equations with delay and a nonlinear source term, J. Nonlinear Sci. Appl., 11 (2018), 17-25. 1, 2, 2, 2

[36] W. S. Zhang, Finite Difference Methods for Patial Differential Equations in Science Computation, Higher Education Press, Beijing, (2006). 4.1 